\title{
FEASIBILITY OF DETERMINING THE MASS OF AN ASTEROID FROM A SPACECRAFT FLYBY
}

\author{
JOHN D. ANDERSON \\ Jet Propu/sion Laboratory
}

\section{APPROXIMATION TO DOPPLER OBSERVABLE}

The orbit of a spacecraft with respect to an asteroid can be approximated to zero order by a hyperbola of zero bending angle (fig. 1). In an orbital system of coordinates $\left(x_{\omega}, y_{\omega}\right)$, the zero-order orbit is given by

$$
\begin{gathered}
x_{\omega}=r^{\prime} \cos f=b \\
y_{\omega}=r^{\prime} \sin f=v(t-T)
\end{gathered}
$$

where $r^{\prime}$ is the distance between the asteroid and the spacecraft, $f$ is the true anomaly, $b$ is the impact parameter or miss distance, $v$ is the constant

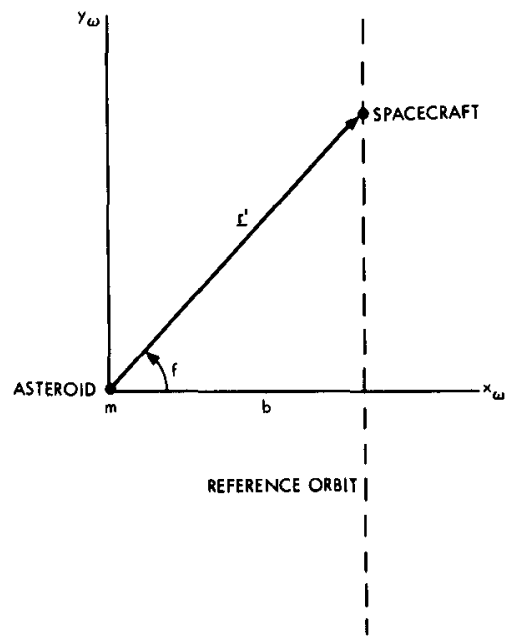

Figure 1.-Geometry of the flyby reference orbit in an orbit-plane coordinate system. The actual orbit is developed to the first order in the mass $m$ as a perturbation from this reference orbit. 
hyperbolic velocity, $t$ is the time of observation, and $T$ is the time of closest approach. After this zero-order solution is substituted into the two-body equations of motion, an approximation to the actual orbit can be obtained. The approximate equations of motion are given by

$$
\begin{aligned}
& \ddot{x}_{\omega}=-\frac{G m b}{r^{\prime 3}} \\
& \ddot{y}_{\omega}=-\frac{G m v(t-T)}{r^{\prime 3}}
\end{aligned}
$$

where

$$
r^{2}=b^{2}+v^{2}(t-T)^{2}
$$

and the results of integration of equations (3) and (4) to the first order in $G m$ are

$$
\begin{aligned}
& \dot{x}_{\omega}=-\frac{G m}{b v} \sin f \\
& \dot{y}_{\omega}=v+\frac{G m}{b v} \cos f
\end{aligned}
$$

where the constants of integration have been chosen such that $v$ is the hyperbolic velocity at infinity. It is not necessary to carry the integration further because the mass $m$ of the asteroid will be determined from Doppler data. Of course, the expressions for $\dot{x}_{\omega}$ and $\dot{y}_{\omega}$ can also be obtained from the hyperbolic orbital equations by establishing approximations for large eccentricities, but the perturbational derivation presented here is slightly easier and more straightforward.

For purposes of determining the mass of an asteroid from the velocity history given by equations (6) and (7), it is sufficient to consider the geocentric range rate $\dot{\Delta}^{\prime}$ to the spacecraft. If the orientation of the spacecraft orbit is referred to the plane of the sky (fig. 2), then the range rate is given by

$$
\dot{\Delta}^{\prime}=\dot{\Delta}-\left(\dot{x}_{\omega} \sin \omega+\dot{y}_{\omega} \cos \omega\right) \sin i+O\left(\frac{r^{\prime}}{\Delta}\right)
$$

(where $O$ means “on the order of magnitude of') and from equations (6) and (7),

$$
\dot{\Delta}^{\prime}=\dot{\Delta}-v \cos \omega \sin i-\frac{G m}{b v} \cos (f+\omega) \sin i+O\left(\frac{r^{\prime}}{\Delta}\right)
$$

Note that the range rate is independent of the location of the node of the spacecraft's orbit on the plane of the sky, but it does depend on the argument of the perifocus and the inclination. 


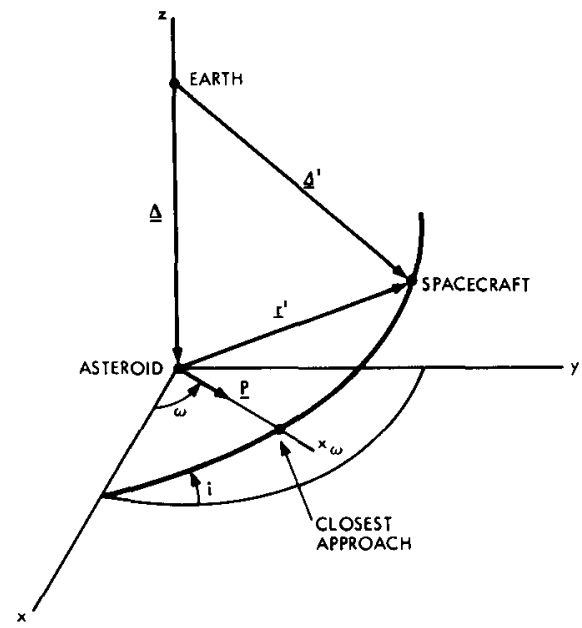

Figure 2.-Geometry of spacecraft encounter with an asteroid in a plane of the sky coordinate system.

However, in a determination of the mass, it is impossible to separate the function $\sin i$ from $\mathrm{Gm}$. Therefore, it is convenient to define a quantity, $G m \sin i$, that can be determined from the range-rate curve. Also, because the zero-order range rate contains no information about the mass of the asteroid, the only term of interest in equation (9) is the one containing $\mathrm{Gm}$. A Doppler observable $z$ that carries all the information on the mass can be defined by

$$
z=-\frac{\zeta}{b v} \cos (f+\omega)
$$

where

$$
\zeta=G m \sin i
$$

\section{COVARIANCE ANALYSIS}

There are a number of unknown parameters in the expression for $z$, but the ones of particular interest to the flyby data are $\zeta, b$, and $T$. The velocity $v$ and the argument of the perifocus $\omega$ can be determined from the ephemerides of the spacecraft and asteroid outside the flyby region, although a reasonably accurate velocity ephemeris for the asteroid is needed for this purpose and also for a determination of the inclination $i$, a quantity required for the separation of $\mathrm{Gm}$ from the mass function $\zeta$.

The sensitivity of $z$ to the three parameters $\zeta, b$, and $T$ can be obtained by partial differentiation, where the true anomaly $f$ is related to $b$ and $T$ by the relation

$$
\tan f=\frac{v}{b}(t-T)
$$


The differentiation yields the following three expressions:

$$
\begin{aligned}
& \frac{\partial z}{\partial \zeta}=-\frac{1}{b v} \cos (f+\omega) \\
& \frac{\partial z}{\partial b}=\frac{\zeta}{b^{2} v}\left(\cos ^{3} f \cos \omega-2 \sin f \sin \omega+\sin ^{3} f \sin \omega\right) \\
& \frac{\partial z}{\partial T}=-\frac{\zeta}{b^{2}} \cos ^{2} f \sin (f+\omega)
\end{aligned}
$$

To construct a covariance matrix on the three parameters, it is necessary to form the products and cross products of the three partial derivatives and then to integrate over $f$ from $-\pi / 2$ to $\pi / 2$, the limits of the zero-order reference orbit. The integration can be performed either with respect to time $t$ or the true anomaly $f$. For the former, an integration with respect to $t$ implies a sampling of the Doppler data at equal intervals of time. With $f$ as the independent variable, the sampling is assumed to occur at equal intervals of the true anomaly. This is probably closer to the actual situation in which it would be expected that the sampling of data would be most frequent around the time of closest approach. Besides, an integration with respect to time results in the same functional form for the standard error on $\zeta$, but the numerical coefficient is smaller than for the integration in the true anomaly. Thus, a sampling at equal intervals of $f$ yields a more conservative estimate of the expected accuracy in $\zeta$ than does a sampling at equal intervals of $t$. In view of all the simplifying assumptions of this analysis, a conservative estimate is preferable.

The integrals of interest are

$$
\begin{gathered}
\int_{-\pi / 2}^{\pi / 2}\left(\frac{\partial z}{\partial \zeta}\right)^{2} d f=\frac{\pi}{2 b^{2} v^{2}} \\
\int_{-\pi / 2}^{\pi / 2}\left(\frac{\partial z}{\partial b}\right)^{2} d f=\frac{\zeta^{2} \pi}{4 b^{4} v^{2}}\left(\frac{9}{4}-\cos 2 \omega\right) \\
\int_{-\pi / 2}^{\pi / 2}\left(\frac{\partial z}{\partial T}\right)^{2} d f=\frac{\zeta^{2} \pi}{8 b^{4}}\left(\frac{3}{2}-\cos 2 \omega\right) \\
\int_{-\pi / 2}^{\pi / 2} \frac{\partial z}{\partial \zeta} \frac{\partial z}{\partial b} d f=-\frac{\zeta \pi}{8 b^{3} v^{2}}(4-\cos 2 \omega)
\end{gathered}
$$




$$
\begin{gathered}
\int_{-\pi / 2}^{\pi / 2} \frac{\partial z}{\partial \zeta} \frac{\partial z}{\partial T} d f=\frac{\zeta \pi}{8 b^{3} v} \sin 2 \omega \\
\int_{-\pi / 2}^{\pi / 2} \frac{\partial z}{\partial b} \frac{\partial z}{\partial T} d f=-\frac{\zeta^{2} \pi}{16 b^{4} v} \sin 2 \omega
\end{gathered}
$$

From equations (20) and (21), it is apparent that at $\omega=0^{\circ}$ and $\omega=90^{\circ}$ the correlation of $T$ with $\zeta$ and $b$ is zero. Therefore, it is reasonable to neglect the correlation with $T$ in all cases, and to compute the two-dimensional covariance matrix on $\zeta$ and $b$. The inverse covariance matrix $\mathbf{J}$ is defined by

$$
\mathbf{J}=\frac{1}{h_{f} \sigma_{z}^{2}} \int_{-\pi / 2}^{\pi / 2}\left(\begin{array}{ll}
\left(\frac{\partial z}{\partial \zeta}\right)^{2} & \frac{\partial z}{\partial \zeta} \frac{\partial z}{\partial b} \\
\frac{\partial z}{\partial \zeta} \frac{\partial z}{\partial b} & \left(\frac{\partial z}{\partial b}\right)^{2}
\end{array}\right) d f
$$

where $h_{f}$ is the sample interval in the true anomaly and $\sigma_{z}$ is the standard error on the Doppler measurements represented by $z$. At the time of closest approach, the sample interval $h_{f}$ in the true anomaly is related to the sample interval $h$ in time by

$$
h_{f}=\frac{v}{b} h
$$

The inverse covariance matrix in terms of $h$ can be obtained by substituting equations (16), (17), and (19) into equation (22).

$$
\mathbf{J}=\frac{\pi}{2 b v^{3} h \sigma_{z}^{2}}\left(\begin{array}{cc}
1 & -\frac{\zeta}{b}(1-1 / 4 \cos 2 \omega) \\
-\frac{\zeta}{b}(1-1 / 4 \cos 2 \omega) & \frac{\zeta^{2}}{2 b^{2}}\left(\frac{9}{4}-\cos 2 \omega\right)
\end{array}\right)
$$

The inverse can be obtained easily, and the standard deviation on $\zeta$ is simply the upper left element of the resulting matrix.

$$
\sigma_{\zeta}^{2}=\frac{4 b v^{3}}{\pi}\left(\frac{9-4 \cos 2 \omega}{2-\cos ^{2} 2 \omega}\right) h \sigma_{z}^{2}
$$

From this expression for the error in the mass function, it can be seen that a better determination of the mass is obtained by viewing the flyby along the orbit path $\left(\omega=0^{\circ}\right)$ rather than across it $\left(\omega=90^{\circ}\right)$. However, the ratio of the 
two standard errors for $\omega=90^{\circ}$ and $\omega=0^{\circ}$ is only $\sqrt{2.6}$, and the sensitivity to $\omega$ is not particularly great. At $\omega=30^{\circ}$, the expression for $\sigma_{\xi}{ }^{2}$ is a minimum, and the resulting value of $\sigma_{\zeta}{ }^{2}$ is about the best that can be expected from the flyby data. This minimum value of $\sigma_{\zeta}^{2}$ is

$$
\sigma_{\zeta}^{2}=\frac{16 b v^{3}}{\pi} h \sigma_{z}^{2}
$$

\section{NUMERICAL RESULTS}

The percentage error in the mass $m$ of the asteroid is related to the error in $\zeta$ by

$$
\frac{\sigma_{m}}{m}=\frac{\sigma_{\zeta}}{\zeta}=\frac{\sigma_{\zeta}}{G m \sin i}
$$

and from equation (26)

$$
\left(\frac{\sigma_{m}}{m}\right)^{2}=\frac{16 b v^{3}}{G^{2} m^{2} \pi} h \sigma_{z}^{2} \csc ^{2} i
$$

The mass $m$ can be replaced by the radius $R$ of the asteroid by means of the relation

$$
m=\frac{4}{3} \pi \rho R^{3}
$$

where $\rho$ is the mean density. The substitution for $m$ can be made in equation (28) and the expression can be rearranged to yield the closest approach distance $b$ as a function of the percentage error in the mass.

$$
b=\frac{\pi^{3}(G \rho)^{2}}{9 h \sigma_{z}^{2}} \frac{R^{6}}{v^{3}}\left(\frac{\sigma_{m}}{m}\right)^{2} \sin ^{2} i
$$

As an estimate of the accuracy in the Doppler data, we will assume that $\sigma_{z}=0.5 \mathrm{~mm} / \mathrm{s}$ and that $h=60 \mathrm{~s}$. This assumption is consistent with the present capability of the NASA/JPL Deep Space Net. In addition, a mean density of $3.33 \mathrm{~g} / \mathrm{cm}^{3}$ will be assumed in the evaluation of equation (30). In units of kilometers and seconds, equation (30) then reduces to

$$
b=1.132 \times 10^{-2} \frac{R^{6}}{v^{3}}\left(\frac{\sigma_{m}}{m}\right)^{2} \sin ^{2} i
$$

or

$$
\log b=-1.946+6 \log R-3 \log v+2 \log \frac{\sigma_{m}}{m}+2 \log (\sin i)
$$


A plot of $\log b$ as a function of $\log R$ is shown in figure 3 for a range of values of $v$ and for a 1 percent determination of the mass. The region of inaccessibility to a flyby $(b \leqslant R)$ is below the dashed line on this plot. The inclination angle is assumed equal to its optimum value of $90^{\circ}$.

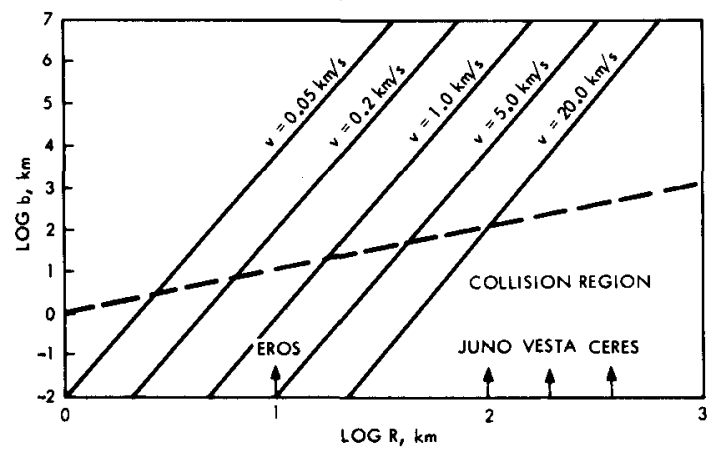

Figure 3.-Miss distance $b$ required to determine the mass of an asteroid of radius $R$ to an accuracy of \pm 1 percent. Curves are shown for various flyby velocities $v$. A density of $3.33 \mathrm{~g} / \mathrm{cm}^{3}$ is assumed in converting from mass to radius. The Doppler data are assumed accurate to $0.5 \mathrm{~mm} / \mathrm{s}$ at a sample rate of one per minute.

Flyby velocities for asteroid missions should be on the order of $5 \mathrm{~km} / \mathrm{s}$. At this speed it should be possible to obtain a 1 percent determination of the masses of the larger asteroids. Ceres would require a flyby distance of $23 \times 10^{6} \mathrm{~km}$, Pallas would require $1.7 \times 10^{6} \mathrm{~km}$, and Vesta, $0.4 \times 10^{6} \mathrm{~km}$. The closest approach to Juno, on the other hand, would have to be on the order of $9100 \mathrm{~km}$ for a 1 percent determination of its mass.

For a smaller asteroid such as Eros, any determination of its mass is practically out of the question for a flyby mission. However, for rendezvous missions with approach speeds less than $1 \mathrm{~km} / \mathrm{s}$, a 10 percent determination of the mass of an asteroid like Eros appears feasible for closest approach distances on the order of a few hundred kilometers. Of course, information on the mass could be destroyed by the performance of terminal maneuvers during the rendezvous.

\section{ACKNOWLEDGMENTS}

The author wishes to thank D. L. Cain and J. Lorell of the Jet Propulsion Laboratory for many helpful discussions on the contents of this paper. This paper presents the results of one phase of research carried out at JPL, California Institute of Technology, under NASA Contract no. NAS 7-100. 